University of Nebraska - Lincoln

DigitalCommons@University of Nebraska - Lincoln

$5-1-2006$

\title{
Non-isothermal reaction-diffusion systems with thermodynamically coupled heat and mass transfer
}

Yasar Demirel

University of Nebraska-Lincoln, ydemirel2@unl.edu

Follow this and additional works at: https://digitalcommons.unl.edu/chemenganalytical

Part of the Chemical Engineering Commons

Demirel, Yasar, "Non-isothermal reaction-diffusion systems with thermodynamically coupled heat and mass transfer" (2006). Papers in Analytical Chemistry. 2.

https://digitalcommons.unl.edu/chemenganalytical/2

This Article is brought to you for free and open access by the Chemical and Biomolecular Engineering Research and Publications at DigitalCommons@University of Nebraska - Lincoln. It has been accepted for inclusion in Papers in Analytical Chemistry by an authorized administrator of DigitalCommons@University of Nebraska - Lincoln. 


\title{
Non-isothermal reaction-diffusion systems with thermodynamically coupled heat and mass transfer
}

\begin{abstract}
Non-isothermal reaction-diffusion (RD) systems control the behavior of many transport and rate processes in physical, chemical, and biological systems. A considerable work has been published on mathematically coupled nonlinear differential equations of RD systems by neglecting the possible thermodynamic couplings among heat and mass fluxes, and reaction velocities. Here, the thermodynamic coupling refers that a flux occurs without its primary thermodynamic driving force, which may be gradient of temperature, or chemical potential, or reaction affinity. This study presents the modeling equations of non-isothermal RD systems with coupled heat and mass fluxes excluding the coupling of chemical reactions using the linear non-equilibrium thermodynamic approach. For a slab catalyst pellet, it shows the dynamic behavior of composition and temperature profiles obtained from the numerical solutions of non-linear partial differential equations by Mathematica for two industrial reaction systems of synthesis of vinyl chloride and dissociation of $\mathrm{N}_{2} \mathrm{O}$.
\end{abstract}

Keywords: Chemical reaction; Heat and mass transfer; Thermodynamic coupling; Nonequilibrium thermodynamics

Comment: This paper was published in the Journal of "Chemical Engineering Science" Volume 61 and Issue 10 on May 2006 Pages 3379-3385, And all the copy rights (C) of this paper belong to Elsevier Ltd. 


\subsection{Introduction}

Modeling of spatio-temporal evolution may serve as a powerful complementary tool to experimental non-isothermal reaction-diffusion (RD) systems within a porous catalyst particle and membrane (Weisz and Hicks, 1962, Hlavecek et al., 1969, Aris, 1975, Froment and Bischoff, 1979, Dekker et al., 1995, Pan and Zhu, 1998, Levent et al., 1998, Levent, 2001, Kafarov et al., 1998 and Burghardt and Berezowski, 2003; Selegny et al., 1995a and Selegny et al., 1995b). Non-isothermal RD systems represent thermodynamically and mathematically coupled transport and chemical processes, and need a through analysis, as they may evolve multiple steady states with unique dynamic phenomena (Burghardt and Berezowski, 2003). Here, the coupling refers that a flux occurs without its primary thermodynamic driving force, or opposite to the direction imposed by its driving force, called the active transport. The principles of thermodynamics allow the progress of a process without or against its primary driving force only if it is coupled with another process. This is consistent with the statement of second law that states that a finite amount of organization may be obtained at the expense of a greater amount of disorganization in a series of coupled spontaneous processes.

Although the change in temperature may be small in a RD system (Weisz and Hicks, 1962 and Froment and Bischoff, 1979), the local gradient of temperature, which is the thermodynamic force, can still induce coupling (Kutchai et al., 1970, Davis, 1991 and Gas et al., 2003). Also, heat effects can enhance the facilitated membrane transport (Selegny et al., 1995a and Selegny et al., 1995b), increase the catalytic membrane activity (Mita, 2000 and Diano et al., 2000), and induce long-range concentration fluctuations in the absence of any convective instability ( $\mathrm{Li}$ et al., 1998 and De Zarate and Sengers, 2004). 


\subsection{Non-isothermal RD systems}

For a steady state, first order $\mathrm{RD}$ system $(A \rightarrow$ product $)$, using a non-isothermal catalyst pellet, and considering molecular transport, the mass and energy balances are

$$
\begin{aligned}
& 0=-\nabla \cdot \mathbf{J}-k_{v} c, \\
& 0=-\nabla \cdot \mathbf{J}_{q}+\left(-\Delta H_{r}\right) k_{v} c .
\end{aligned}
$$

By using the Fick and Fourier laws in one-dimensional transport in a slab catalyst pellet with, equimolar counter diffusion under mechanical equilibrium, Eqs. (1) and (2) become

$$
\begin{aligned}
& 0=D_{e} \frac{\mathrm{d}^{2} c}{\mathrm{~d} x^{2}}-k_{v} c, \\
& 0=k_{e} \frac{\mathrm{d}^{2} T}{\mathrm{~d} x^{2}}+\left(-\Delta H_{r}\right) k_{v} c .
\end{aligned}
$$

Without the external mass and heat transfer resistances, the boundary conditions with $\mathrm{x}$ coordinate oriented from the centerline $(x=0)$ to the surface $(x=L)$ are

$$
c(L)=c_{s}, \quad \frac{\mathrm{d} c(0)}{\mathrm{d} x}=0 \quad \text { and } \quad T(L)=T_{s}, \quad \frac{\mathrm{d} T(0)}{\mathrm{d} x}=0 .
$$

Here $\Delta H_{r}$ is the heat of reaction, and $D_{e}$ and $k_{e}$ are the effective diffusivity and thermal conductivity, respectively, and tend to be less than the ordinary gas diffusivity and the thermal conductivity for a non-porous similar solid (Pan and Zhu, 1998). The effective reaction rate $\left(k_{v} c\right)$ is based on the total rate of reaction within any small, representative volume.

Eliminating the reaction terms from Eqs. (3) and (4), and integrating twice with the boundary conditions given in Eq. (5), temperature is related to concentration by 


$$
\begin{aligned}
& \varphi=1-\beta(\theta-1), \\
& \text { where } \\
& \theta=\frac{c}{c_{s}}, \quad \varphi=\frac{T}{T_{s}}, \quad \beta=\frac{\left(-\Delta H_{r}\right) D_{e} c_{s}}{k_{e} T_{s}} .
\end{aligned}
$$

Eq. (6) is valid for any particle geometry under steady-state conditions, and can be used to eliminate $\theta$ or $\$$ from one of the differential equations $\underline{(3)}$ and (4).

The value of $\beta$ is a measure of non-isothermal effects; for increasing values of $\beta$, nonisothermal effects may become important (Froment and Bischoff, 1979 and Tavera, 2005). Heat of reaction is strongly nonlinear function of temperature. For any interior points within the pore where the reactant is largely consumed, the maximum temperature difference for an exothermic reaction becomes

$$
\Delta T_{\max }=\left(T-T_{s}\right)_{\max }=\frac{\left(-\Delta H_{r}\right) D_{e} c_{s}}{k_{e}}=\beta T_{s} .
$$

Using the dimensionless parameters

$$
\begin{aligned}
& z=\frac{x}{L}, \quad \tau=\frac{D_{e} t}{L^{2}}, \quad \phi^{2}=\frac{L^{2} k_{0} \exp \left(E / R T_{s}\right)}{D_{e}}, \\
& \gamma=\frac{E}{R T_{s}}, \quad L e=\frac{k_{e} / \rho C_{p}}{D_{e}}=\frac{\alpha_{e}}{D_{e}}
\end{aligned}
$$

the transient forms of Eqs. (3) and (4) become

$$
\frac{\partial \theta}{\partial \tau}=\frac{\partial^{2} \theta}{\partial z^{2}}-\phi^{2} \theta \exp [\gamma(1-1 / \varphi)],
$$

where $L e$ is the modified Lewis number, and $\underline{\alpha}_{\underline{e}}$ is the effective thermal diffusivity. The initial and boundary conditions are

$$
\begin{aligned}
& \theta(0, z)=1, \quad \theta(\tau, 1)=1, \quad \mathrm{~d} \theta(\tau, 0) / \mathrm{d} z=0, \\
& \varphi(0, z)=1, \quad \varphi(\tau, 1)=1, \quad \mathrm{~d} \varphi(\tau, 0) / \mathrm{d} z=0 .
\end{aligned}
$$


After using Eq. (6) in Eq. (8), steady and non-isothermal concentration profile becomes

$$
\frac{\mathrm{d}^{2} \theta}{\mathrm{d} z^{2}}=\phi^{2} \theta \exp \left(-\frac{\gamma \beta(\theta-1)}{1-\beta(\theta-1)}\right) .
$$

\subsection{RD systems with thermodynamically coupled heat and mass transfer}

Non-isothermal RD systems represent open, non-equilibrium systems with thermodynamic forces of temperature gradient, chemical potential gradient, and affinities. In a non-equilibrium system, temperature and internal energy are well defined in an elemental volume containing a sufficient number of molecules. Therefore a local equilibrium exists, and the thermodynamic properties are related to the state variables in the same manner as in equilibrium (Prigogine, 1967, Wisniewski et al., 1976, Kondepudi and Prigogine, 1999, Demirel, 2002 and Demirel and Sandler, 2004); the total entropy $S$ and energy $U$ are expressed by entropy $s$ and energy $u$ densities:

$$
\begin{gathered}
S=\int_{V} s\left[T(x), n_{i}(x)\right] \mathrm{d} V . \\
U=\int_{V} u\left[T(x), n_{i}(x)\right] \mathrm{d} V
\end{gathered}
$$

and hence the local variables become $(\partial S / \partial U)_{n i}=1 / T(x)$ and $\left(\partial S / \partial N_{i}\right)_{u}=-\mu(x) / T(x)$. Mostly, the internal relaxation processes in a fluid or material are much faster than the rate of change imposed upon the state variables, and the local equilibrium concept is valid for a wide range of transport and rate processes of usual fluid systems (Sieniutycz, 1992, Kondepudi and Prigogine, 1999, Demirel and Sandler, 2001 and Demirel and Sandler, 2004).

The rate of entropy production $\Phi$ due to irreversible processes is always positive, and calculated in terms of conjugate forces $X$ and fluxes $J$ as

$$
\Phi=\left(d_{i} S / \mathrm{d} V \mathrm{~d} t\right)=\sum J_{k} X_{k} \geqslant 0
$$

within a system. For a multicomponent fluid system under mechanical equilibrium with $n$ species and $N_{r}$ number of chemical reactions, the dissipation function $\Psi$ is obtained from 
the general balance equations including the entropy balance and the Gibbs relation ( $\underline{\mathrm{De}}$ $\underline{\text { Groot and Mazur, 1985, 1985; Wisniewski et al., } 1976 \text { and Demirel and Sandler, 2001) }}$

$$
\begin{aligned}
\Psi & =T \Phi \\
& =\left(-\frac{1}{T} \mathbf{J}_{q} \nabla T-\sum_{i=1}^{n} \mathbf{J}_{i}\left(\nabla \mu_{i}\right)+\sum_{j=1}^{N_{r}} J_{r j} A_{j}\right) \geqslant 0
\end{aligned}
$$

where, $\mathbf{J}_{q}$ is the vector of reduced heat flux (Demirel and Sandler, 2001), and $\mathbf{J}_{i}$ is the vector of mass fluxes, $\mu_{i}$ is the chemical potential of species $i, A$ is the affinity $A=-\sum v_{i} \mu_{i}, v$ is the stoichiometric coefficients, and $J_{r}$ is the reaction velocity. Eq. (13) consists of scalar processes of chemical reactions and vectorial processes of heat and mass transfer, while it excludes viscous flow flux, electrical, and magnetic effects.

In the dissipation-phenomenological equation (DPE) approach (Demirel and Sandler, 2001), Eq. (13) identifies a set of independent conjugate fluxes $J$ and forces $X$ to be used in the following linear phenomenological equations when system is not far away from global equilibrium:

$$
J_{i}=\sum_{k=1}^{m} L_{i k} X_{k}
$$

The phenomenological coefficients $L_{i k}(i, k=1,2, \ldots, m)$ can be determined experimentally (Rowley, 1989 and Demirel and Sandler, 2002). The values of $L_{i k}$ satisfy that $L_{i i} L_{k k^{-}}$ $L_{i k} L_{k i} \geqslant 0$ and $L_{i i} \geqslant 0 ; L_{k k} \geqslant 0$, and Onsager's reciprocal relations state that the cross (coupling) coefficients are symmetric $L_{i k}=L_{k i} ;(i \neq k)$ for a set of independent conjugate fluxes and forces identified by the dissipation function or the rate of entropy production (Wisniewski et al., 1976, Kondepudi and Prigogine, 1999, Demirel and Sandler, 2001 and Demirel, 2002). Onsager's relations are based on microscopic reversibility, and are independent of the state of a system or any other microscopic assumptions (De Groot and Mazur, 1985; Kondepudi and Prigogine, 1999). 
Excluding the coupling of chemical reaction with the heat and mass transfer, and using $\nabla$ $\mu_{1}=\left(\partial \mu_{1} / \partial c_{1}\right) \nabla c_{1}$, we identify the fluxes and forces for a binary mixture from Eq. (13)

$$
\Psi=-\frac{1}{T} \mathbf{J}_{q} \nabla T-\mathbf{J}_{1} \lambda_{T, P} \nabla c_{1} \geqslant 0,
$$

where

$$
\lambda_{T, P}=\left(1+\frac{\bar{V}_{1 c_{1}}}{\bar{V}_{2 c 2}}\right)\left(\frac{\partial \mu_{1}}{\partial c 1}\right)_{T, P},
$$

and $\bar{F}_{\text {lis }}$ the partial molar volume of component $i$. Assuming that the RD system is not far from global equilibrium, the linear phenomenological equations based on Eq. (15) are

$$
\begin{aligned}
& -\mathbf{J}=L_{11} \lambda_{T, P} \nabla c+\frac{1}{T} L_{1 q} \nabla T=D_{e} \nabla_{c}+D_{S, e} \nabla T, \\
& -\mathbf{J}_{q}=L_{q 1} \lambda_{T, P} \nabla c+\frac{1}{T} L_{q q} \nabla T=D_{D, e} \nabla c+k_{e} \nabla T,
\end{aligned}
$$

where $D_{D, e}$ is a coefficient in $\mathbf{m}^{2} \mathbf{J} /$ ( $\left.\mathbf{m o l} \mathbf{~ s}\right)$ related to the effective Dufour effect, and $D_{S, e}$ is a coefficient in $\mathrm{mol} /(\mathrm{m} \mathrm{s} \mathrm{K})$ related to the effective Soret effect (thermal diffusion). When there is no volume flow, the mass flux $\mathbf{J}_{1}$ is: $\mathbf{J}=-L_{11} \lambda_{T, P} \nabla c$, and comparing it with Fick's law $\mathbf{J}=-D_{e} \nabla c$, the coefficient $L_{11}$ is related to the effective diffusion coefficient by $L_{11}=D_{e} / \lambda_{T, P}$. Using Fourier's law $\mathbf{J}_{q}=-k_{e} \nabla T$ in Eq. (17), the primary coefficient $L_{q q}$ is related to the effective thermal conductivity $k_{e}$ by $L_{q q}=k_{e} T$. The thermal diffusion coefficient for species 1 is $D_{T}=L_{1 q} /(c T)$. For liquids the diffusion coefficient $D$ is of the order of $10^{-5} \mathbf{c m}^{2} / \mathbf{s}$, and the thermal diffusion coefficient $D_{T}$ is of the order of $\mathbf{1 0}^{-8}-\mathbf{1 0}^{-10}$ $\mathbf{c m}^{2} / \mathbf{s}$. For gases the order of magnitude for $D$ is $\mathbf{1 0}^{-1} \mathrm{~cm}^{2} / \mathbf{s}$ and $10^{-4}-\mathbf{1 0}^{-6} \mathrm{~cm}^{2} / \mathbf{s}$, respectively (Wisniewski et al., 1976).

By using the fluxes $\mathbf{J}_{i}$ and $\mathbf{J}_{q}$ from Eqs. (16) and (17), respectively in Eqs. (1) and (2), and with the coupled values of temperature $T^{\prime}$ and concentration $c^{\prime}$, we have

$$
\begin{aligned}
& 0=\nabla \cdot\left(D_{e} \nabla c^{\prime}+D_{S, e} \nabla T^{\prime}\right)-k_{v} c^{\prime}, \\
& 0=\nabla \cdot\left(D_{D, e} \nabla c^{\prime}+k_{e} \nabla T^{\prime}\right)+\left(-\Delta H_{r}\right) k_{v} c^{\prime} .
\end{aligned}
$$


As before, elimination of the reaction terms from Eqs. (18) and (19) yields

$$
\varphi^{\prime}=1-\beta^{\prime}(\theta-1),
$$

where

$$
\theta^{\prime}=\frac{c^{\prime}}{c_{s}}, \quad \varphi^{\prime}=\frac{T^{\prime}}{T_{s}} \quad \text { and } \quad \beta^{\prime}=\frac{\left(D_{e}\left(-\Delta H_{r}\right)+D_{D, e}\right) c_{s}}{\left(k_{e}+D_{S, e}\left(-\Delta H_{r}\right)\right) T_{s}}
$$

and the maximum temperature difference becomes

$$
\beta^{\prime} \approx\left(\varphi_{\text {center }}-1\right)=\frac{\Delta T_{\max }^{\prime}}{T_{s}} .
$$

The modified dimensionless group $\beta^{\prime}$ represents the ratio between the rate of heat of chemical reaction and the rate of heat conduction when the heat and mass fluxes are coupled only. By neglecting the coupling effects, we would have $\beta^{\prime}=\beta$.

Using Eqs. (18) and (19) with the Arrhenius equation $k_{v}=k_{0} \mathrm{e}^{-E / R T}$ we have the transient forms of the coupled heat and mass fluxes for a single component

The initial and boundary conditions are the same with those given in Eqs. (8) and (9). For a simple plane geometry and one-dimensional unsteady state equimolar diffusion, Eqs. (23) and (24) become

$$
\begin{gathered}
\frac{\partial c^{\prime}}{\partial t}=\nabla \cdot\left(D_{e} \nabla c^{\prime}+D_{S, e} \nabla T^{\prime}\right)-\left(k_{0} \mathrm{e}^{-E / R T^{\prime}}\right) c^{\prime}, \\
\begin{array}{c}
\rho C_{p} \frac{\partial T^{\prime}}{\partial t}=\nabla \cdot\left(D_{D, e} \nabla c^{\prime}+k_{e} \nabla T^{\prime}\right) \\
+\left[-\left(\Delta H_{r}\right)\right]\left(k_{0} \mathrm{e}^{-E / R T^{\prime}}\right) c^{\prime} .
\end{array}
\end{gathered}
$$




$$
\begin{aligned}
& \frac{\partial \theta^{\prime}}{\partial \tau}=\frac{\partial^{2} \theta^{\prime}}{\partial z^{2}}+\varepsilon \frac{\partial^{2} \varphi^{\prime}}{\partial z^{2}}-\phi^{2} \theta^{\prime} \exp \left[\gamma\left(1-\frac{1}{\varphi^{\prime}}\right)\right], \\
& \frac{1}{L e} \frac{\partial \varphi^{\prime}}{\partial \tau}=\frac{\partial^{2} \varphi^{\prime}}{\partial z^{2}}+\omega \frac{\partial^{2} \theta^{\prime}}{\partial z^{2}}+\beta^{\prime} \phi^{2} \theta^{\prime} \exp \left[\gamma\left(1-\frac{1}{\varphi^{\prime}}\right)\right],
\end{aligned}
$$

where

$$
\varepsilon=\frac{D_{S, e} T_{s}}{D_{e} c_{s}}, \quad \omega=\frac{D_{D, e} c_{s}}{k_{e} T_{s}}
$$

All the other non-dimensional parameters are the same with those in Eqs. (8) and (9). Eqs. (25) and (26) represent the modeling of RD systems with the thermodynamically coupled heat and mass fluxes excluding the coupling effects due to reaction when compared with Eqs. (8) and (9). After combining Eq. (20) with Eqs. (25) and (26), steady-state balance equations for a RD system with the coupled heat and mass transfer become

$$
\begin{aligned}
& \left(1-\beta^{\prime} \varepsilon\right) \frac{\mathrm{d}^{2} \theta^{\prime}}{\mathrm{d} z^{2}}=\phi^{2} \theta^{\prime} \exp \left(-\frac{\gamma \beta^{\prime}\left(\theta^{\prime}-1\right)}{1-\beta^{\prime}\left(\theta^{\prime}-1\right)}\right), \\
& \left(\frac{\omega}{\beta^{\prime}}-1\right) \frac{\mathrm{d}^{2} \varphi^{\prime}}{\mathrm{d} z^{2}}=\beta^{\prime} \phi^{2}\left(1-\frac{\varphi^{\prime}-1}{\beta^{\prime}}\right) \exp \left[\gamma\left(1-\frac{1}{\varphi^{\prime}}\right)\right] .
\end{aligned}
$$

Since the dynamic behavior of a RD system may be more apparent with the state-space diagrams, the temperature and concentration profiles are replaced with the spatial integral averages obtained from

$$
\begin{aligned}
& \theta^{\prime}(\tau)=\int_{0}^{1} \theta^{\prime}(z, \tau) \mathrm{d} z \\
& \varphi^{\prime}(\tau)=\int_{0}^{1} \varphi^{\prime}(z, \tau) \mathrm{d} z .
\end{aligned}
$$




\subsection{Results}

Non-isothermal reaction diffusion systems considered are the synthesis of vinyl chloride and dissociation of $\mathrm{N}_{2} \mathrm{O}$ reactions. Table 1 shows some of the experimental and assumed values of the parameters (Hlavecek et al., 1969) considered for these reaction systems, which have large values of $\beta$, and hence display relatively strong non-isothermal effects. The thermal diffusion coefficient is usually smaller by a factor of $10^{2}-10^{3}$ than the ordinary diffusion coefficient for non-electrolytes and gases. Therefore, for the present analysis the values for $\varepsilon$ and $\omega$ are assumed to be 0.001 for the both reaction systems. The Mathematica is used to solve the partial differential equations of mathematically and thermodynamically coupled systems of Eqs. (25) and (26) simultaneously. The numerical solutions from Mathematica are obtained with a 'precision goal' of 0.1 , 'maximum step size' of 0.0005 , and 'maximum steps' of infinity.

\section{Table 1.}

Experimental (Hlavecek et al., 1969) and assumed values of parameters used in the thermodynamically coupled model for the two industrial reaction systems

\begin{tabular}{|l|l|l|l|l|l|l|}
\hline Reaction & $\boldsymbol{\varphi}$ & $\boldsymbol{\beta} \approx \boldsymbol{\beta}^{\prime}$ & $\boldsymbol{\gamma}$ & $\boldsymbol{L} \boldsymbol{e}$ & $\boldsymbol{\varepsilon}^{\mathbf{a}}$ & $\boldsymbol{\omega}^{\mathbf{a}}$ \\
\hline Synthesis of vinyl chloride & 0.27 & 0.25 & 6.5 & 0.1 & 0.001 & 0.001 \\
\hline Dissociation of $\mathrm{N}_{2} \mathrm{O}$ & 5.0 & 0.64 & 22.0 & $0.01^{\underline{\mathrm{a}}}$ & 0.001 & 0.001 \\
\hline
\end{tabular}

${ }^{\mathrm{a}}$ Assumed values.

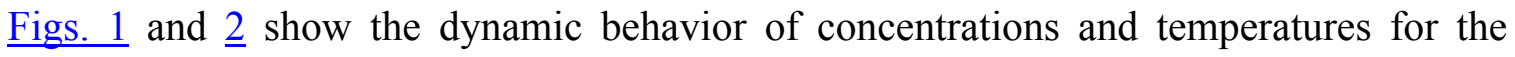
synthesis of vinyl chloride reaction and dissociation of $\mathrm{N}_{2} \mathrm{O}$ reaction, respectively. In both the reaction systems, the surface of temperature closely follows the change in concentrations. As expected, the rise of temperature is relatively larger as the value of $\beta$ increases in dissociation of $\mathrm{N}_{2} \mathrm{O}$ reaction as seen in Fig. 2. 
Fig. 1 Dynamic behavior of thermodynamically coupled non-isothermal reaction-diffusion system of diffusion system of synthesis of vinyl chloride reaction: (a) concentration surface, (b) temperature surface. The parameters used are in Table1
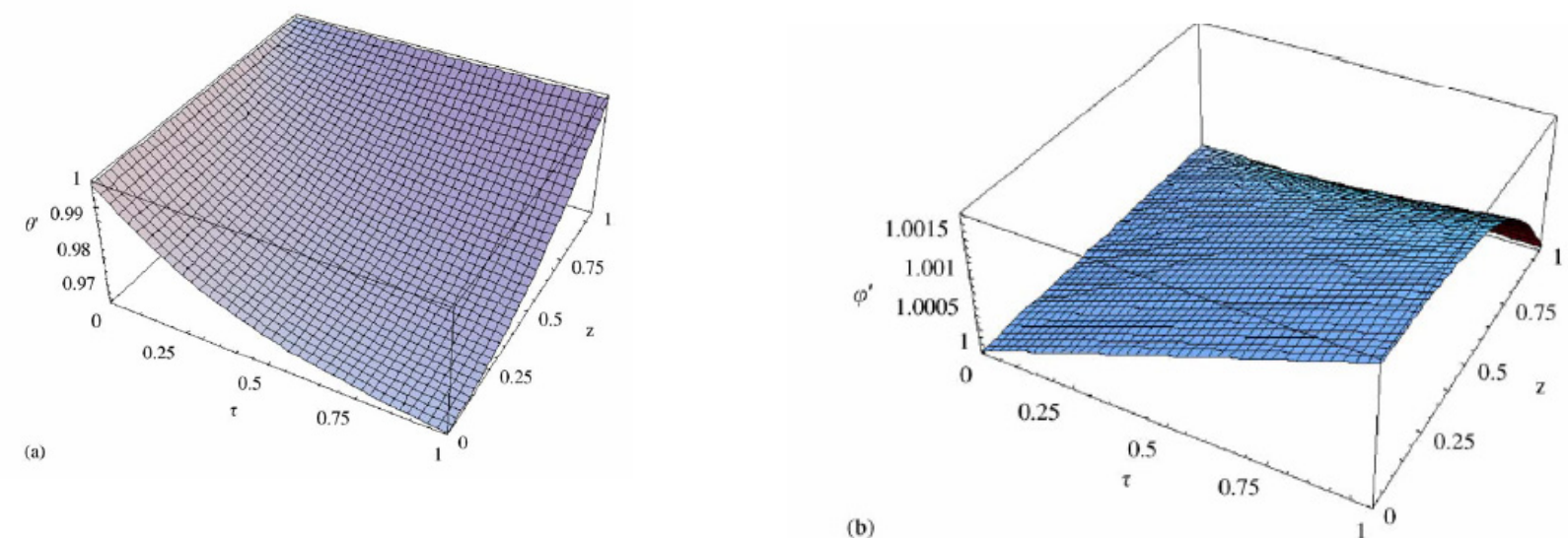

Fig. 2. Dynamic behavior of thermodynamically coupled non-isothermal reactiondiffusion system of dissociation of $\mathrm{N}_{2} \mathrm{O}$ reaction: (a) concentration surface, (b) temperature surface. The parameters used are in Table 1.

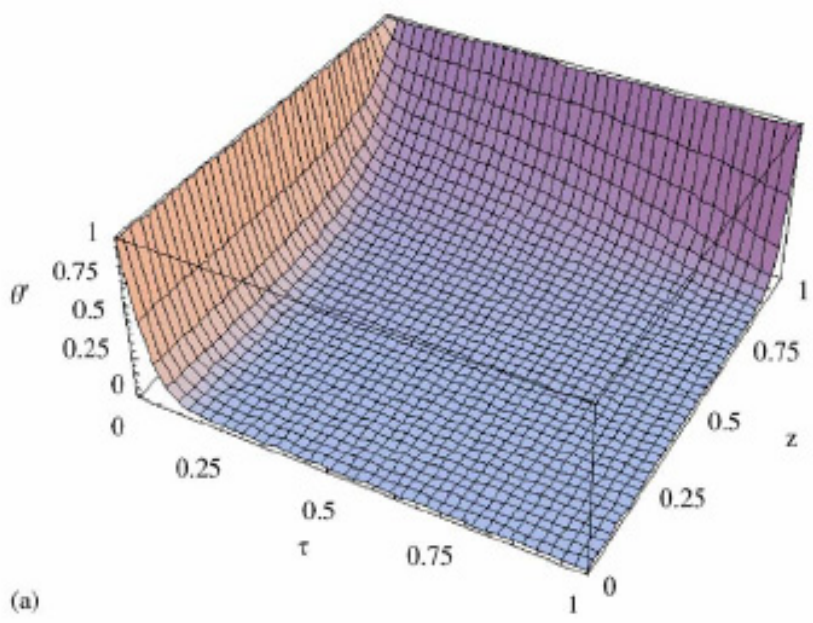

(a)

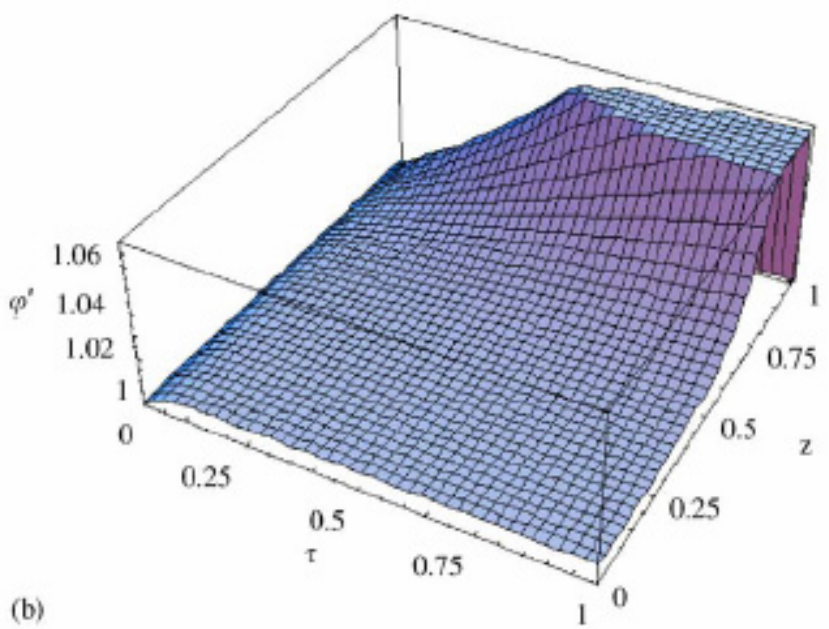


Figs. 3 and 4 show the changes of the spatial integral averages of concentration and temperatures with time for the systems considered. Figs. 3(c) and $\underline{4}$ (c) show the statespace representation of temperature versus concentration when the time changes from zero to one. For the synthesis of vinyl chloride reaction system, temperature reaches its maximum value when the dimensionless concentration is around 0.97 , as seen in 3 (c)
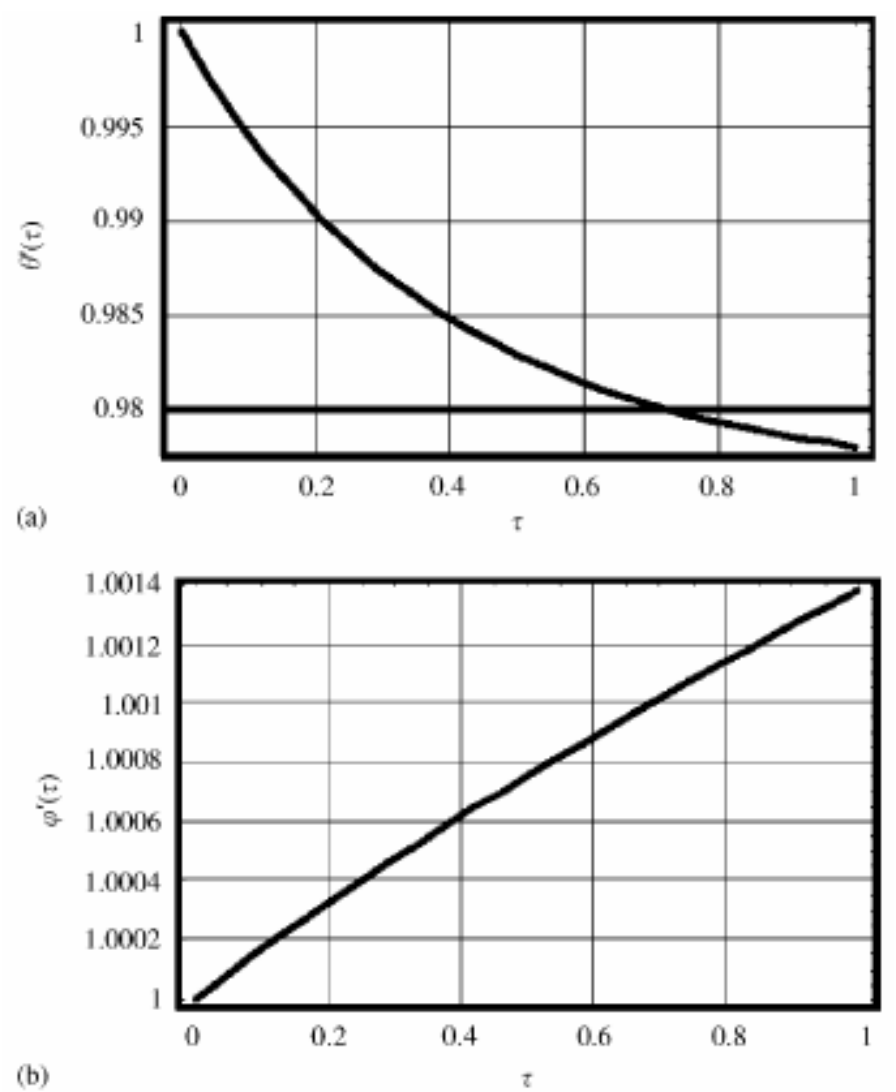

(b)

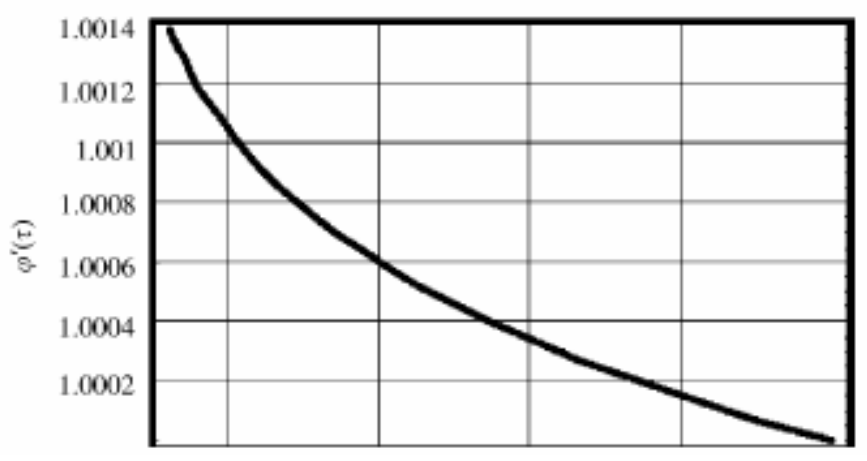


Fig. 3. Spatial integral averages for the synthesis of vinyl chloride reaction system: (a) change of concentration with time, (b) change of temperature with time, (c) change of temperature with concentration when the time varies between 0 and 1 . The parameters used are in Table 1 .
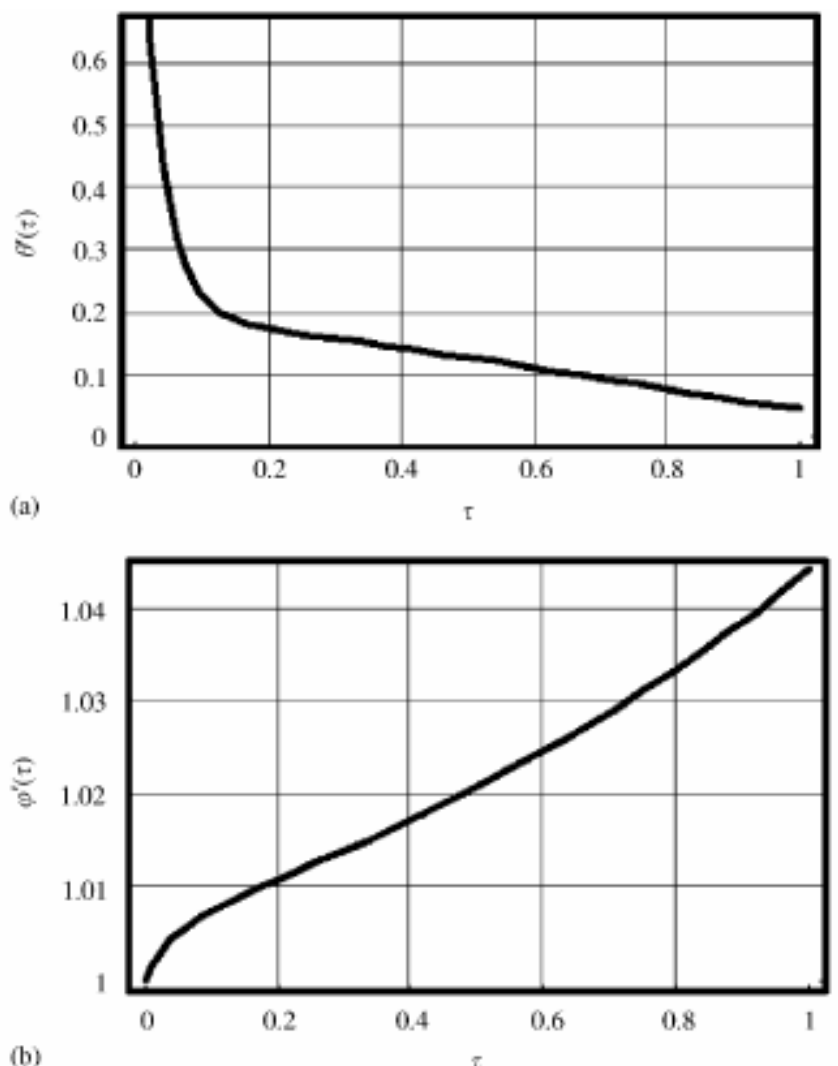

(b)

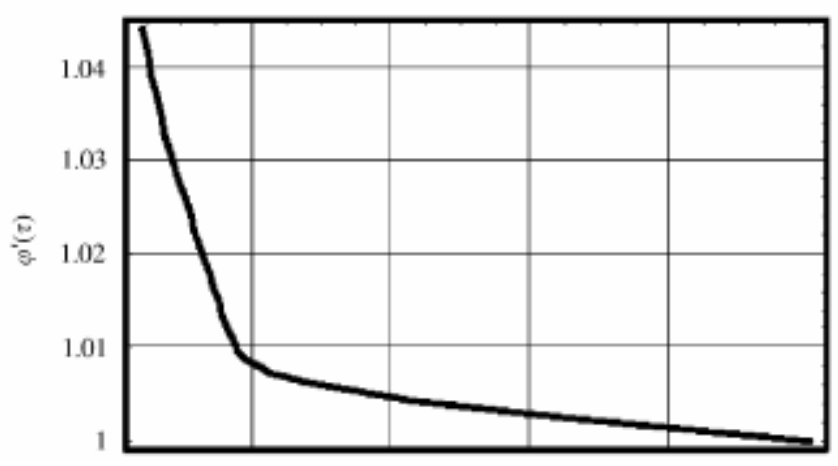

Fig. 4. Spatial integral averages for synthesis of dissociation of $\mathrm{N}_{2} \mathrm{O}$ reaction system: (a) change of concentration with time, (b) change of temperature with time, (c) change of temperature with concentration when the time varies between 0 and 1 . The parameters used are in Table 1. 


\subsection{Conclusions}

This analysis considers the thermodynamic coupling between heat and mass fluxes in the two industrial RD systems of synthesis of vinyl chloride and dissociation of $\mathrm{N}_{2} \mathrm{O}$ reaction systems. The modeling with the coupling effects of Soret and Dufour would open the path to describe more complex RD systems by adding the two new controlling parameters into their dynamic behavior. The linear non-equilibrium thermodynamics approach can provide the quantified description of the fully coupled phenomena for systems not far from global equilibrium. 


\section{References}

Aris, 1975 Aris, R., 1975. The Mathematical Theory of Diffusion and Reaction in Permeable Catalysts. The Theory of the Steady State, vol. 1. Clarendon Press, Oxford.

Burghardt and Berezowski, 2003 A. Burghardt and M. Berezowski, Periodic solutions in a porous catalyst pellet-homoclinic orbits, Chemical Engineering Science 58 (2003), pp. 2657-2670. Abstract

Davis, 1991 S. Davis, Unsteady facilitated transport of oxygen in hemoglobin-containing membranes and red cells, Journal of Membrane Science 56 (1991), pp. 341-358. Abstract

De Groot and Mazur, 1985 De Groot, S.R., Mazur, P., 1985. Non-equilibrium Thermodynamics. Dover, London.

Dekker et al., 1995 F.H.M. Dekker, A. Bliek, F. Kapteijn and Moulijn, Analysis of mass and heat transfer in transient experiments over heterogeneous catalyst, Chemical Engineering Science 50 (1995), pp. 3573-3580. Abstract

Demirel, 2002 Demirel, Y., 2002. Non-equilibrium Thermodynamics: Transport and Rate Processes in Physical and Biological Systems. Elsevier, Amsterdam, pp. 234-257.

Demirel and Sandler, 2001 Y. Demirel and S.I. Sandler, Linear non-equilibrium thermodynamics theory for coupled heat and mass transport, International Journal of Heat and Mass Transfer 44 (2001), pp. 2439-2451. Abstract

Demirel and Sandler, 2002 Y. Demirel and S.I. Sandler, Effects of concentration and temperature on the coupled heat and mass transport in liquid mixtures, International Journal of Heat and Mass Transfer 45 (2002), pp. 75-86. $\underline{\text { Abstract }}$

Demirel and Sandler, 2004 Y. Demirel and S.I. Sandler, Nonequilibrium thermodynamics in engineering and science, Journal of Physical Chemistry B 108 (2004), pp. 31-43. $\underline{\text { Abstract-Compendex }} \mid \underline{\text { Full Text via CrossRef }}$ 
De Zarate and Sengers, 2004 O.J.M. De Zarate and J.V. Sengers, On the physical origin of long-ranged fluctuations in fluids in thermal nonequilibrium states, Journal of Statistical Physics 115 (2004), pp. 1363-1381.

Diano et al., 2000 N. Diano, M.M. El-Marsy, M. Portaccio, M. Santucci, A. De Maio, V. Grano, D. Castagnolo, U. Bencivenga, F.S. Gaeta and D.G. Mita, The process of thermodialysis and the efficiency increase of bioreaction operating under non-isothermal conditions, Journal Molecular Catalysis B. Enzymatic 11 (2000), pp. 97-111.

Froment and Bischoff, 1979 F.G. Froment and K.B. Bischoff, Chemical Reactor Analysis and Design, Wiley, New York (1979).

Gas et al., 2003 P. Gas, C. Girardeaux, D. Mangelinck and A. Portavoce, Reaction and diffusion at interfaces of micro- and nanostructured materials, Materials Science and Engineering B 101 (2003), pp. 43-48. $\underline{\text { Abstract }}$

Hlavecek et al., 1969 H. Hlavecek, M. Kubicek and M. Mareek, Analysis of nonstationary heat and mass transfer in a porous catalyst particle I, Journal of Catalysis 15 (1969), pp. 31-42.

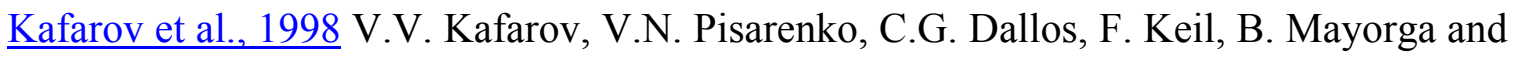
M. Nunez, New method for computer aided analysis of steady-state multiplicity of catalytic processes, Computers in Chemical Engineering 22 (1998) (Suppl.), pp. S659S662. Abstract

Kondepudi and Prigogine, 1999 Kondepudi, D., Prigogine, I., 1999. Modern Thermodynamics; From Heat Engines to Dissipative Structures. Wiley, New York, NY, pp. 351-366.

Kutchai et al., 1970 H. Kutchai, J.A. Jacquez and F.J. Matner, Non-equilibrium facilitated transport in hemoglobin solution, Biophysics Journal 10 (1970), pp. 38-54. Abstract-MEDLINE 
Levent, 2001 M. Levent, Water-gas shift reaction over porous catalyst: temperature and reactant concentration distribution, International Journal of Hydrogen Energy 26 (2001), pp. 551-558. $\underline{\text { Abstract }}$

Levent et al., 1998 M. Levent, G. Budak and A. Karabulut, Estimation of concentration and temperature profiles for methane-steam reforming reaction in a porous catalyst, Fuel Processing Technology 55 (1998), pp. 251-263. $\underline{\text { Abstract }}$

Li et al., 1998 W.B. Li, K.J. Zhang, J.V. Sengers, R.W. Gammon and J.M.O. Zarate, Concentration fluctuations in a polymer solution under a temperature gradient, Physical Review Letters 81 (1998), pp. 5580-5583. Abstract-INSPEC | Full Text via CrossRef | APS full text

Mita, 2000 D.G. Mita, Biocatalytic membrane operating under conditions: fundamentals and applications, Recent Advances in Biotechnology Bioengineering 1 (2000), pp. 1-16.

Pan and Zhu, 1998 T. Pan and B. Zhu, Study of diffusion-reaction process inside a cylindrical pellet, Chemical Engineering Science 53 (1998), pp. 933-946. Abstract

Prigogine, 1967 Prigogine, I., 1967. Introduction to Thermodynamics of Irreversible Processes, third ed. Wiley, New York.

Rowley, 1989 R.I. Rowley, Measurements of heats of transport in ternary liquid mixtures via the diffusion thermoeffect, Journal of Non-Equilibrium Thermodynamics 14 (1989), pp. 293-297.

Selegny et al., 1995a E. Selegny, J.N. Ghogomu, R. Roux, D. Langevin and M. Metayer, Carrier-mediated, thermal energy-coupled, non-isothermal membrane transport. Part I, Transfer of the solute boric acid. Journal of Membrane Science 108 (1995), pp. 161-169. $\underline{\text { Abstract }}$

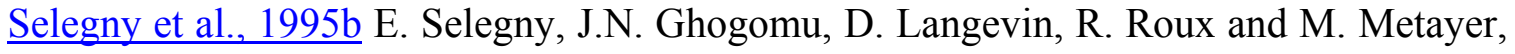
Carrier-mediated, thermal energy-coupled, non-isothermal membrane transport. II. A gaseous system, Journal of Membrane Science 108 (1995), pp. 161-169. Abstract 
Sieniutycz, 1992 Sieniutycz, S., 1992. Wave equations of heat and mass transfer. In: Sieniutycz, S., Salamon, P. (Eds.), Flow, Diffusion, and Rate Processes. Taylor \& Francis, New York, pp. 146-167.

Tavera, 2005 E.M. Tavera, Analytical expression for the effectiveness factor: the nthorder reaction in a slab geometry, Chemical Engineering Science 60 (2005), pp. 907-916. $\underline{\text { Abstract-Compendex }}|\underline{\text { Abstract-FLUIDEX }}| \underline{\text { Abstract-INSPEC }}$

Weisz and Hicks, 1962 P.B. Weisz and J.S. Hicks, The behavior of porous catalyst pellet in view of internal mass and heat diffusion effects, Chemical Engineering Science 41 (1962), pp. 179-186.

Wisniewski et al., 1976 S. Wisniewski, B. Staniszewski and R. Szymanik, Thermodynamics of Non-equilibrium Processes, D. Reidel Publishing Company, Dordrecht 